\title{
Zustandsüberwachung der Statorisolation von umrichtergespeisten elektrischen Maschinen mittels transienter Stromcharakteristik
}

\author{
C. Zöller, M. A. Vogelsberger, T. M. Wolbank OVE
}

\begin{abstract}
In dieser Arbeit wird ein Konzept für die Zustandsüberwachung der Statorisolation von elektrischen Maschinen anhand der transienten Stromauswertung beschrieben. In elektrischen Traktionsanwendungen wird der Motor über einen Wechselrichter betrieben, welcher die Speisung aus dem Zwischenkreis mit variabler Frequenz und Amplitude liefert. Dabei stellen Motor und Umrichter getrennte Funktionseinheiten dar, welche separat entworfen und über Zuleitungen miteinander gekoppelt werden. Die Gesamtstruktur spaltet sich in die Hauptkomponenten Umrichter, Kabel und Maschine. Nach der Beschreibung der Komponenten und deren Wechselwirkungen werden das Prinzip der Überwachungsmethode präsentiert und die Eigenschaften anhand von Messungen an einer Testmaschine verifiziert. Versuche mit künstlich gealterten Wicklungssystemen beschreiben die Zusammenhänge zwischen Änderungen der transienten Signalverläufe und dem Verlust der Isolationsfestigkeit im Wicklungssystem.
\end{abstract}

Schlüsselwörter: umrichtergespeiste Antriebe; Isolationszustandsüberwachung; Isolationsalterung

Stator insulation monitoring of inverter-fed machines based on current transient evaluation.

In this paper, a concept for the condition monitoring of the stator insulation of electrical machines is described using the transient current evaluation. In electrical traction applications, the motor is operated via a voltage source inverter which supplies the machine with voltage variable in frequency and amplitude. The inverter-fed drive system can be described in principle by three main components: the inverter, the cabling and the machine, which define a complex impedance system. The motor and inverter represent separate functional units, which are designed separately. Inverter and machine are connected via the supply lines. If a critical cable length is reached reflection phenomena and overvoltages occur. After describing the components and their interactions, the principle of the monitoring method is presented and verified on a test machine. Experiments with artificially aged winding systems describe the relationships between the change in the transient current signals and the loss of the insulation properties in the winding system.

Keywords: inverter-fed drives; insulation monitoring; insulation aging; transients

Eingegangen am 3. November 2017, angenommen am 20. Februar 2018, online publiziert am 15. März 2018

๑ The Author(s) 2018. Dieser Artikel ist auf Springerlink.com mit Open Access verfügbar

\section{Einleitung}

Die Relevanz von Systemen zur Zustandsüberwachung der Komponenten drehzahlvariabler elektrischer Antriebe, nimmt durch die immer wichtigere Thematik der Elektromobilität ständig zu. Speziell im Hinblick auf die Bahnindustrie sind Sicherheitsstandards und Zuverlässigkeit entscheidende Faktoren. Eine wesentliche Komponente im elektrischen Antrieb stellt der Traktionsmotor dar, welcher leistungsstark sowie kostengünstig ausgelegt werden muss. Grundsätzlich entscheidet die Auslegung wie fehlertolerant bzw. fehlersicher eine Maschine, ein Antrieb oder ein System ist. Die erwartete bzw. geforderte Betriebsdauer liegt bei derzeitigen Traktionsmaschinen im Bereich mehrerer Jahrzehnte. Der Wartungsaufwand in diesem Zeitraum soll so gering wie möglich sein um Kosten zu senken. Dazu wird die kontinuierliche Zustandsüberwachung zur frühzeitigen Fehlererkennung und Ausfallsvermeidung eingesetzt. Durch den Einsatz elektronischer Diagnosesysteme, welche den Zustand einzelner Komponenten kontinuierlich überwachen können sich anbahnende Schäden frühzeitig erkannt werden. Aufwandsintensive Instandhaltungsmaßnahmen mit starrer Zuordnung zu Laufleistungsgrenzen können infolgedessen durch vorausschauende Instandhaltungsprozesse ersetzt werden. Mit dem Übergang von zeitbasierten zu zu- standsorientierten Wartungs- und Instandhaltungsplänen, können sowohl Kosten als auch Aufwand drastisch reduziert werden.

Kernpunkt einer zustandsorientierten Wartung ist eine genaue Zustandsüberwachung. Ein Versagen der Wicklungsisolation wird wegen seiner unmittelbaren und dramatischen Auswirkungen auf den Betrieb des gesamten Antriebs als besonders kritisch angesehen, weshalb der Überwachung des Zustandes der Statorisolation ein besonderer Stellenwert bei der Betrachtung der Zuverlässigkeit von Antrieben eingeräumt wird.

Die hier vorgestellte Methode der Statorisolationsüberwachung basiert auf der Auswertung der transienten Stromcharakteristik unmittelbar nach einer Schalthandlung des speisenden Umrichters. Die Alterung der Betriebsmittel führt zu einer Verschlechterung der Iso-

Zöller, Clemens, Institut für Energiesysteme und elektrische Antriebe, Technische Universität Wien, Gußhausstraße 25-29, 1040 Wien, Österreich

(E-Mail: clemens.zoeller@tuwien.ac.at); Vogelsberger, Markus A., Product \& RuT_Management RoQ_Drives Rolling Stock Equipment (RoQ_Drives), Bombardier Transportation Austria GmbH, Hermann Gebauer-Straße 5, 1220 Wien, Österreich; Wolbank, Thomas M., Institut für Energiesysteme und elektrische Antriebe, Technische Universität Wien Gußhausstraße 25-29, 1040 Wien, Österreich 


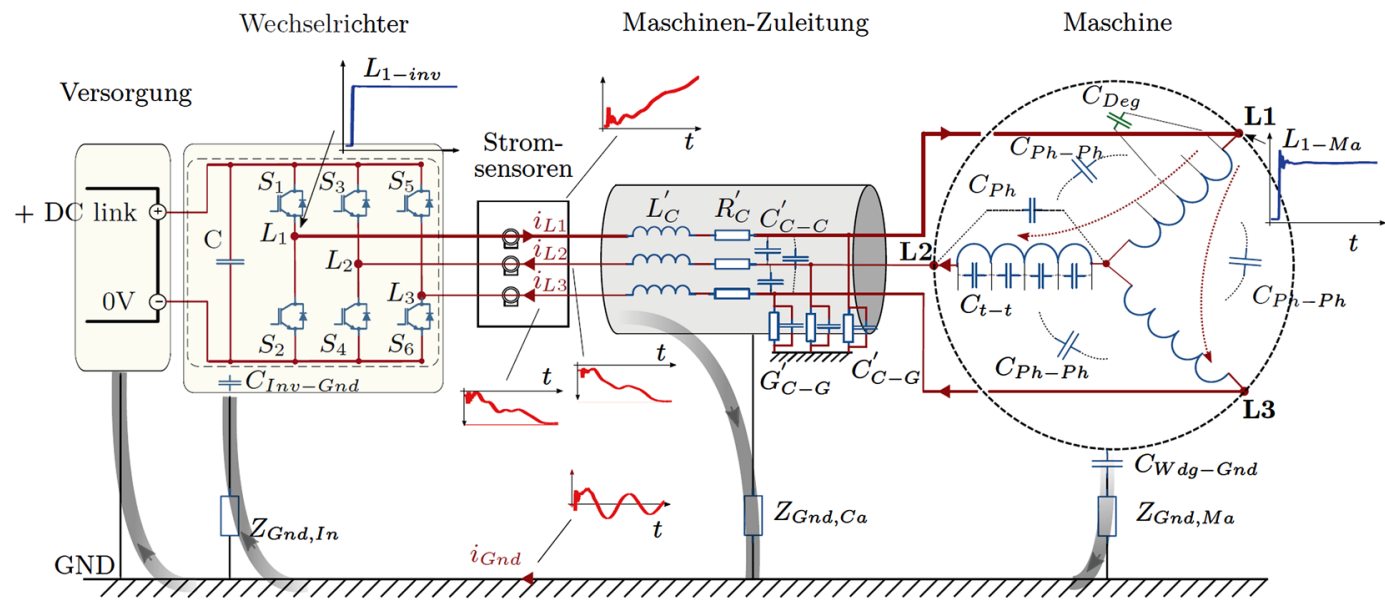

Abb. 1. Prüfstand mit Hauptkomponenten Umrichter, Kabel und Maschine

lationseigenschaften. Neben der dauerhaften Abnahme der elektrischen Festigkeit, wird auch speziell das Hochfrequenzverhalten der Maschine beeinflusst. Diese Eigenschaft und die daraus entstehenden Charakteristika werden bei pulsweitenmodulierter Ansteuerung der Maschine durch den Wechselrichter genutzt. Die Pulsanregung bewirkt eine hochfrequente Reaktion des Systems. Dabei liegen vor allem Effekte im Fokus, die durch unvermeidbare parasitäre Größen, allen voran die inneren Kapazitäten der Maschine sowie Kapazitäten gegen das Maschinengehäuse etc., bestimmt sind. Durch eine Änderung der Isolationsfestigkeit werden auch diese parasitären Eigenschaften verändert und daraus folgend wird in der hier vorgestellten Methode auf eine Isolationszustandsänderung geschlossen.

Im Normwesen sind einige Verfahren definiert, um die Auswirkungen verschiedener Belastungen auf die Isolation festzustellen. Damit soll auf eine Alterung bzw. Veränderung der Betriebsmittel geschlossen werden. Die Ursachen von Belastungen können in mechanische, elektrische, thermische oder umweltbedingte Einflüsse/Belastungen eingeteilt werden. Beispiele für in Normen verankerte Alterungsprüfverfahren für elektrische Traktionsmaschinen sind etwa IEC 6003418-31 bzw. IEEE 1776. Die in diesen Normen festgeschriebenen Prüfverfahren sind grundsätzlich vergleichsbasierende Verfahren zur Qualifizierung von Isoliersystemen. Bei Diagnoseverfahren zur Feststellung der aktuellen Isolationsfestigkeit, z. B. Verlustfaktormessung bzw. Kapazitätsmessung sowie Teilentladungsmessungen wird basierend auf Vergleichsmessungen auf eine Verschlechterung der Isolationsfestigkeit geschlossen. Die genannten Verfahren gehören zu den wichtigsten Methoden der dielektrischen Diagnostik. Für die Durchführung der Messungen wird prinzipiell der gesamte Antrieb außer Betrieb genommen. In den meisten Fällen erfolgt zusätzlich auch die Trennung zwischen Maschine und Umrichter. Dies stellt jedoch erheblichen Aufwand dar.

Ziel war es daher, eine Methode zu entwickeln bei welcher die Maschine ohne Demontage des Antriebs und während des Betriebs oder zumindest in Phasen des Stillstands auf Veränderung im Isolationssystem getestet werden kann. Ein System zur Zustandsüberwachung darf jedoch auch möglichst keine zusätzlichen Kosten verursachen. Daher können nur die Signale bereits im Antrieb verbauter Sensoren für die Zustandsüberwachung eingesetzt werden.

Das Prinzip des Verfahrens und die Eigenschaften werden in den nächsten Kapiteln anhand von Versuchsreihen an einem Prüfstand näher erläutert.

\section{Prüfstand und Messmethode}

In Abb. 1 ist der Prüfstand bestehend aus den Hauptkomponenten Wechselrichter, Kabel und Maschine dargestellt. Die Anregung des Systems durch eine Schalthandlung der Halbbrücke verbunden mit Phase L1 der Maschine ist angedeutet.

Beim Wechselrichter handelt es sich um einen 3-phasigen 2-level Spannungszwischenkreis-Wechselrichter. Diese Konfiguration stellt den einfachsten Aufbau dar und wird im Bahnbereich hauptsächlich verwendet. Die Zuleitung mit einer Länge von etwa $10 \mathrm{~m}$ verbindet Wechselrichter und Maschine. Als Maschine dient eine Asynchronmaschine (ASM) mit Kurzschlussläufer und einer Leistung von 1,4 MW, welche als Traktionsmaschine konzipiert wurde. Zusätzlich sind die signifikanten elektrischen Komponenten, sowie parasitär wirkende Komponenten eingezeichnet. Im Falle des Wechselrichters handelt es sich um die parasitäre Kapazität $C_{G N D}$ und die Impedanz $Z_{G N D, n}$. Die Verkabelung zwischen Wechselrichter und Maschine wird durch das verlustbehaftete Modell für Übertragungsleitungen repräsentiert, mit den Parametern Widerstand $R_{C}^{\prime}$, Induktivität $L_{C}^{\prime}$, Kapazitäten $C_{C-C}^{\prime}$ und $C_{C}^{\prime}$ sowie Leitfähigkeit $G_{C}^{\prime}$ pro Längeneinheit. Innerhalb der Maschine wirken neben den Grundwellenparametern (Statorwiderstand, Statorinduktivität) die parasitären Wicklungsparameter, dargestellt mit der Kapazität zwischen Wicklung und dem geerdeten Statorgehäuse $C_{G n d}$, der Phase zu Phase-Kapazität $C_{P h-P h}$, der Phasenkapazität $C_{P h}$ und die Windung zu Windung Kapazität $C_{t-t}$.

Beim Betrieb von elektrischen Maschinen an einem Wechselrichter entstehen, bedingt durch die Speisung mittels Pulsweitenmodulation (PWM), Belastungen einzelner Komponenten im System durch parasitäre kapazitive Spannungen und Ströme, welche auch in der Abb. 1 angedeutet werden. Der Stromlaufpfad während einer Schalthandlung des Wechselrichters ist angedeutet und teilt sich in den niederfrequenten Strom über die Zuleitung und Maschinenwicklung $\left(i_{L 1}, i_{L 2}\right.$ und $\left.i_{L 3}\right)$, sowie den hochfrequenten Anteil, welcher kapazitiv über Kabelschirmung und Gehäuse mit Erdschiene/Leitung gekoppelt ist und sich über den Erdpfad wieder mit dem Wechselrichter bzw. Versorgung schließt. Die Erdströme des Systems sind durch $i_{G N D}$ dargestellt, wobei der wesentliche Teil des Erdstroms über die Statorwicklungskapazitäten und das Motorgehäuse fließt. Daneben sollen auch noch die bei den elektrischen Maschinen hochfrequenten Lagerströme erwähnt werden, die hier nicht eingezeichnet sind und sehr verschleißfördernd wirken. In [1] wird der Einfluss dieser Parameter auf die Signalverläufe anhand eines HochfrequenzModells der Statorwicklung von elektrischen Maschinen untersucht. 


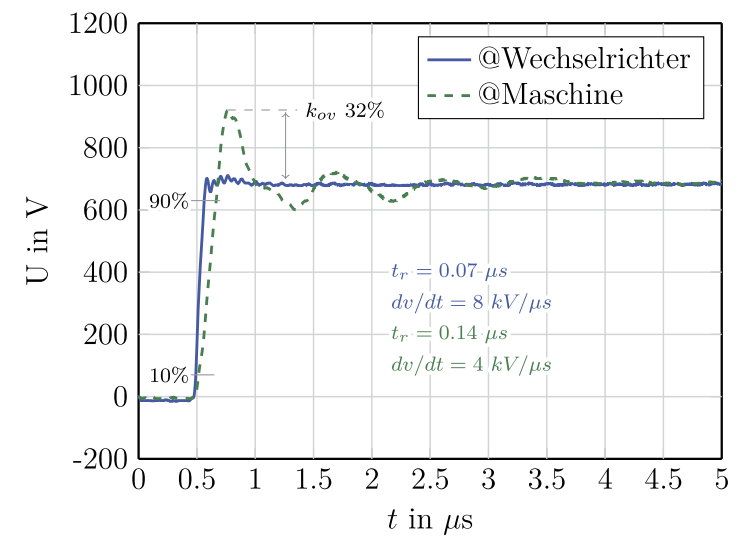

Abb. 2. Spannung am Wechselrichterausgang und an der Maschine bezogen auf das negative Zwischenkreispotential

Die transienten Ströme werden hierbei vor allem durch die inneren Kapazitäten beeinflusst, welche sich durch geometrisch und Material bedingte Kapazitäten der Windungen ergeben und der allgemeinen Wicklungskapazität zum Maschinengehäuse (Ständer und Läufer). Die Höhe der kapazitiven Ströme ergibt sich durch den Spannungsgradienten der speisenden Spannung und der Größe der Kapazitäten $\left(i_{c}=C d U / d t\right)$. Bei hohen Kapazitätswerten von einigen Nanofarad ergeben sich bereits kapazitive Ströme von einigen Ampere.

Die Spannung, direkt gemessen am Wechselrichterausgang und an der Maschine, bezogen auf das negative Zwischenkreispotential ist in Abb. 2 dargestellt. Die Anstiegszeit der Ausgangspannung einer Wechselrichter-Halbbrücke beträgt in diesem Fall $t_{r} \sim 70 \mathrm{~ns}$. Als Grenzen für den Bereich der Anstiegszeit wurden die Werte zwischen $10 \%$ und $90 \%$ des stationären Endwerts herangezogen. Dadurch ergibt sich ein Spannungsgradient $d u / d t$ von etwa $8 \mathrm{kV} / \mu \mathrm{s}$ am Wechselrichterausgang. Dies stellt einen typischen Wert für einen Traktionsantrieb im Mittelspannungsbereich dar. Selbst IGBT-Wechselrichter erreichen in heutigen Anwendungen bereits ein $d u / d t$ von etwa 10-15 kV/ $\mu \mathrm{s}$. Im Prüfstandsaufbau wird zusätzlich auch ein Wechselrichter basierend auf moderneren SiC-MOSFET Leistungshalbleitern verwendet, mit welchem ein Spannungsgradient von bis zu 25 kV/Ms erreicht werden kann. Durch die ständige Weiterentwicklung im Bereich der Leistungshalbleiter in Richtung höherer Schaltfrequenz und Flankensteilheit, wird der Anteil der parasitären Effekte immer weiter erhöht.

Der zeitliche Verlauf der Spannung an der Maschine ist strichliert in der Abb. 2 dargestellt. Auffallend hierbei ist der weniger steile Anstiegsverlauf im Gegensatz zum Wechselrichterausgang. Für Spannungspulse mit hoher Flankensteilheit stellt die Wicklung der Maschine mit ihrer hauptsächlichen induktiven und ohmschen Eigenschaft im Zusammenhang mit den parasitären Kapazitäten, z. B. Erdkapazität, einen Tiefpass dar. Dadurch dringen hochfrequente Anteile nicht in die Wicklung ein, sondern werden reflektiert und erzeugen in den Eingangsspulen der Wicklung hohe und schnell abklingende kapazitive Überspannungen.

Gleichzeitig steigt aber auch die Belastung des Isolationssystems durch reflexionsbedingte Überspannungen am Leitungsende an der Maschine, wie in der Abbildung durch den Überspannungsfaktor $k_{\text {ov }}$ mit $32 \%$ zu sehen ist, und auf Grund der nichtlinearen Spannungsverteilung längs der Wicklungsstränge. Letztere bedeutet auch eine höhere Belastung vor allem für die ersten Spulen eines Stranges.
Reflexionen am Leitungsende an der Maschine sind eine Folge der nicht vorhandenen Impedanzanpassung zwischen Zuleitungskabel und Maschine. Anhand der Theorie werden Reflexionen nach dem einfachen Leitungsmodell einer nicht ideal angepassten Signalleitung beschrieben mit dem Reflexionskoeffizienten $\Gamma$, bestimmt durch die fehlende Impedanzanpassung des Leitungs-Maschinen Paares und mit der kritischen Kabellänge $I_{c}$, ab welcher mit der höchst anzunehmenden Überspannung zu rechnen ist, cf. [2, 3]. Das Zusammenspiel von langer Motorzuleitung, steilen Spannungsflanken und Impedanzanpassung wird durch die Gl. (1a)(1d) beschrieben. Die Impedanzanpassung wird mit (1a) dem Wellenwiderstand beschrieben, wobei hier Reflexion und Resonanzeffekte durch das Verhältnis von Induktivitäts- und Kapazitätsbelag der Verbindungsleitungen vom Wechselrichter zur Maschine (Wellenwiderstand $Z_{\text {cable }}$ ) und dem Wellenwiderstand der Maschine (Wellenwiderstand $Z_{\text {mot }}$ ) entstehen. Durch die Spannungsanstiegszeit $t_{r}$ am Wechselrichterausgang, sowie der Ausbreitungszeit bzw. -geschwindigkeit der elektromagnetischen Welle $t_{p}, v_{p}$ kann die kritische Kabellänge, ab welcher eine volle Reflexion der eintreffenden Spannungswelle erfolgt, beschrieben werden. Dadurch ergibt sich die Gl. (1b), welche die kritische Kabellänge beschreibt. GI. (1c) zeigt, dass die Ausbreitungsgeschwindigkeit von der längenbezogenen Leitungsinduktivität sowie Leitungskapazität abhängig ist. Mit Gl. (1d) ergibt sich ab erreichen der kritischen Kabellänge die resultierende Spannung an der Maschine $U_{m a}$ erhöht durch den Reflexionskoeffizienten $\Gamma$.

$$
\begin{gathered}
\Gamma_{m o t}=\frac{\left(Z_{m o t}-Z_{\text {cable }}\right)}{\left(Z_{m o t}+Z_{\text {cable }}\right)} \\
t_{r}=2 t_{p}=2 \frac{I_{\text {cable }}}{v_{p}} \quad b z w . \quad I_{\text {cable, } c r i t}=v_{p} \frac{t_{r}}{2} \\
v_{p}=\frac{1}{\sqrt{L_{C}^{\prime} \cdot C_{C}^{\prime}}} \\
U_{m a}=U_{d c}+\Gamma_{m o t} U_{d c}
\end{gathered}
$$

Im Folgenden soll das Prinzip der Überwachung des Isolationszustandes genauer behandelt werden.

\section{Verifikation der Isolationseigenschaften basierend auf transienter Auswertung}

Im Hinblick auf die Anwendung in industriellen Antrieben, wird im weiteren Verlauf nur die Stromantwort analysiert, da in den meisten Fällen aus Kostengründen keine Spannungssensoren in einem industriellen Traktionsantrieb eingesetzt werden. Die Charakteristik der transienten Stromantwort hängt hauptsächlich von den parasitären Kapazitäten, gebildet aus dem Wicklungssystem in Kombination mit der Isolation und dem Gehäuse, ab.

In Abb. 3 werden zwei, in der Phase L1 gemessene, Stromantworten nach einer Anregung durch einen Spannungssprung verglichen. Die Anregung erfolgt durch einer Schalthandlung des Wechselrichters, wobei vom Ausgangszustand mit allen Phasen am niederen Zwischenkreis (ZK) - Spannungspotential, in der entsprechenden Phase ein Wechsel zum höheren ZK-Potential erfolgt. Beide Kurvenverläufe stellen die transiente Stromreaktion auf die Spannungsanregung an derselben Maschine dar, jedoch repräsentiert das Signal, gekennzeichnet mit „Initialmessung” die unveränderte gesunde Maschine und das Signal bezeichnet mit „emulierte Alterung” zeigt eine Änderung des Isolationszustands. Eine Veränderung der Isolationsfestigkeit wird mit parallel zum Wicklungssystem angeordnetem Kondensator emuliert. Dies wird durch den speziell für diese Untersuchungen entworfenen Mittelspannungs-Traktionsmotor 


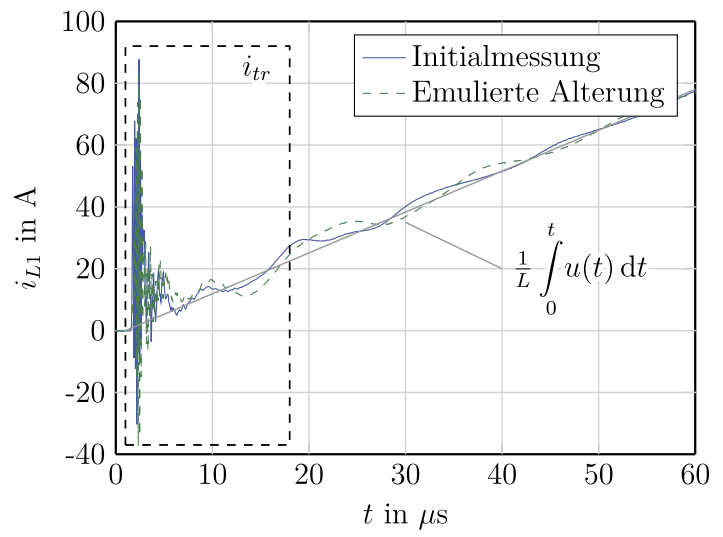

Abb. 3. Transiente Stromantworten nach Schalthandlung des Wechselrichters in Phase L1

(1,4 MW) mit Windungsanzapfungen ermöglicht, cf. Abb. 1, $\Delta \mathrm{C}$. Die Änderung der gesamten Wicklungskapazitäten der Maschine ist kennzeichnend für den Verlust der Isolationsfestigkeit. Dieses Verhalten kann in beschleunigten Alterungstests, wie in Abschn. 4 beschrieben, nachgewiesen werden.

Der Einschwingvorgang der Stromantworten in Abb. 3 klingt nach wenigen $\mu$ stark ab und geht in den linearen Stromanstieg über, bestimmt durch das hauptsächlich induktive Verhalten der Maschine. Jedes einzelne aufgezeichnete Signal wird als Überlagerung eines linearen Stromanstiegs $i_{L}(t)$ (aufgrund der induktiven Eigenschaften der Maschine) und des transienten hochfrequenten Teils $i_{t r}(t)$ gesehen. Dies ist mit Gl. (2) beschrieben.

$$
i(t)=i_{t r}(t)+i_{L}(t)=i_{t r}(t)+\frac{1}{L} \int_{0}^{t} u(t) d t
$$

Der lineare Stromanstieg hängt von der Maschineninduktivität und den inhärenten Asymmetrien ab, u.a. Nutung oder Sättigung. Der transiente Teil ist deutlich von der Kapazitätsänderung abhängig, cf. Abb. 3. Die Signalanteile werden durch geeignete Verfahren voneinander getrennt, um im weiteren Verlauf die Änderung der Isolationseigenschaften anhand der transienten Stromantwort genauer untersuchen zu können. Des Weiteren sollen Einflüsse wie etwa die Rotorposition die Ergebnisse nicht beeinflussen.

Durch die Änderung der Wicklungskapazitäten wird das Hochfrequenzverhalten der Maschine deutlich beeinflusst. Durch die Abweichung der transienten Oszillation von einer zu Beginn der Lebensdauer gemachten Referenzmessung, kann auf eine Zustandsänderung geschlossen werden. Basierend auf der Änderung der Frequenzkomponenten der transienten Stromantwort wird eine Abweichung vom Bekannten Isolationszustand als eine anormale Signalcharakteristik eingestuft. Abbildung 4 zeigt die Spektralkomponenten der transienten Stromantworten, „Initialmessung" - unveränderte gesunde Maschine zu Beginn der Lebensdauer und "emulierte Alterung" - emulierte Änderung des Isolationszustands. Es zeigt sich deutlich, dass eine Abweichung über einen weiten Frequenzbereich entsteht.

Deutlich zu erkennen sind die Abweichungen im Bereich bis etwa $200 \mathrm{kHz}$. Oberhalb von $200 \mathrm{kHz}$ nehmen die Spektralanteile deutlich $a b$, jedoch ist noch immer eine Abweichung zwischen "Initialmessung" und "emulierter Alterung" vorhanden. Welche Spektralanteile zwischen bekanntem Initialzustand und emulierter Alterung variieren, ist abhängig von Position bzw. Größe des Kondensators, welcher durch Windungsanzapfungen parallel zur Wicklung der Maschine eingebracht werden kann, cf. Abb. $1 \Delta C$.

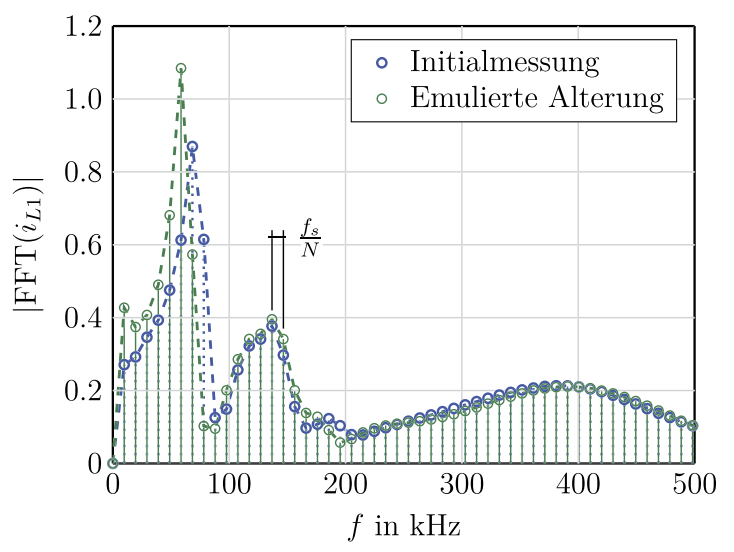

Abb. 4. Fourieranalyse des transienten Stromverlaufs

In einem letzten Verfahrensschritt wird die Abweichung mit einem Indikator ausgedrückt um den Schweregrad der Abweichung anzugeben und eine Interpretation der Ergebnisse zu vereinfachen. Als einfache und elegante Variante für einen Indikator wurde das Fehlerquadrat zwischen den Amplitudenspektren und den äquidistanten Frequenzpunkten, siehe Gl. (3) ausgewählt.

$$
\begin{aligned}
\text { Indikator }_{p, k} & =\operatorname{std}\left(\bar{Y}_{p, \text { ref }}, Y_{p, a k t, k}\right) \\
& =\sqrt{\frac{\sum_{i=1}^{n}\left(\bar{Y}_{p, r e f}\left(f_{i}\right)-Y_{p, a k t, k}\left(f_{i}\right)\right)^{2}}{n}} \\
\text { mit } \bar{Y}_{p, \text { ref }}\left(f_{i}\right) & =\frac{\sum_{k=1}^{m} Y_{p, \text { ref }, k}\left(f_{i}\right)}{m}
\end{aligned}
$$

Das Zeitsignal der Referenzmessung wird in Form der spektralen Anteile mit der Bezeichnung $\bar{Y}_{p, \text { ref }}$ repräsentiert, wobei der Querstrich auf den Mittelwert von mehreren Messungen, definiert durch den Wert $m$, hindeutet. Der Index $p$ bezeichnet die untersuchte bzw. ausgewertete Phase ( $L 1, L 2$ oder $L 3)$. Jede weitere aktuell aufgenommene Messung an der Maschine wird mit $Y_{p, a k t, k}\left(f_{i}\right)$ bezeichnet. Der zusätzliche Index $k$ steht für die Nummer der aktuell durchgeführten Messung. Die Variable $n$ ist abhängig von der gewählten Breite des zu betrachtenden Frequenzbereichs, definiert durch die Festlegung einer unteren bzw. oberen Grenzfrequenz $n=f_{\text {low }}-f_{\text {high }}$. Durch die Definition und Auswahl eines abgegrenzten Frequenzbereiches für die Indikator-Berechnung lassen sich Störeffekte, bedingt durch Zuleitung, Schirmungen etc. ausblenden. Darüber hinaus können Störungen auch durch eine einfache Gewichtung $g=g_{1}, g_{2} \ldots g_{n}$ der einzelnen äquidistanten Frequenzkomponenten $f_{1}, f_{2} \ldots f_{n}$ vermieden werden, wie in Gl. (4) zu sehen ist.

$$
\begin{aligned}
\text { Indikator }_{k} & =s t d\left(\bar{Y}_{p, \text { ref }}, Y_{p, a k t, k}\right) \\
& =\sqrt{\frac{\sum_{i=1}^{n}\left(\bar{Y}_{p, \text { ref }}\left(f_{i}\right)-Y_{p, a k t, k}\left(f_{i}\right)\right)^{2} \cdot g_{i}}{n_{g}}}
\end{aligned}
$$

Um die Vergleichbarkeit zu gewährleisten, ist zusätzlich noch der Frequenzbereich mit der Variable $n_{g}$ anzupassen. Dieser Einfluss resultiert oft aufgrund von spezifischen Charakteristika der Sensoren.

Da eine Einzelmessung nur wenige hundert Mikrosekunden benötigt, werden in einem Messzyklus mehrere Messungen herangezogen um einen Zustand der Maschine zu beschreiben. Dadurch ergeben sich verschiedene statistische Methoden zur Auswertung und Interpretation der Ergebnisse. Die Einzelindikatoren der Phasen 


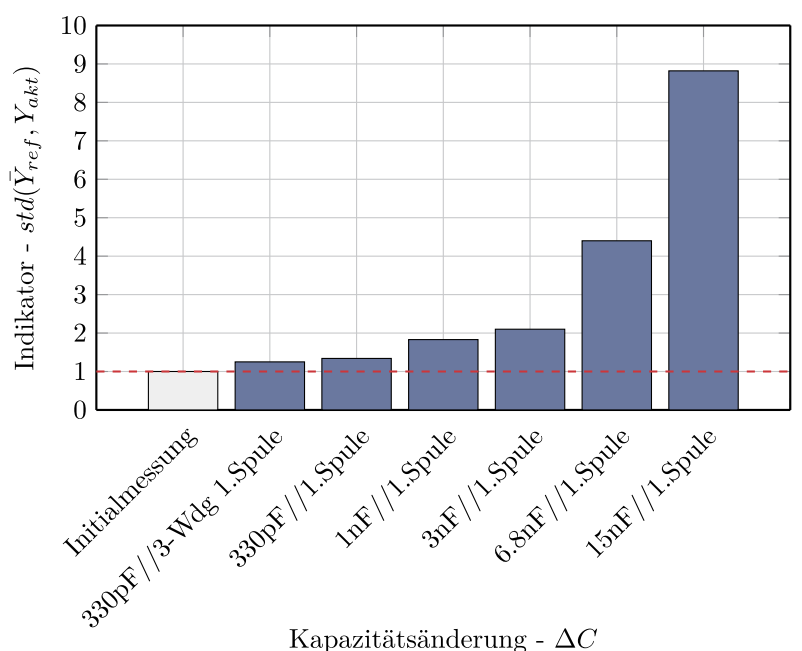

Abb. 5. Veränderung des Isolationszustandsindikators bei emulierter Isolationszustandsveränderung

Indikator $_{p, k}$ werden durch Mittelwertbildung nach Gl. (5)

$$
\text { Indikator }_{p}=\frac{\sum_{k=1}^{m} \text { Indikator }_{p, k}}{m}
$$

zu einem Gesamtindikator der Phase und sind für verschiedene emulierte Szenarien im Balkendiagramm (Abb. 5) dargestellt. Die Werte sind normiert auf die Abweichung innerhalb der Initialmessung. Somit ergibt sich aus einem Wert größer als 1 eine Änderung vom Initialzustand. Eine grundsätzliche Fragestellung betrifft die Art und Weise, wie die Alterung bzw. Schwächung der Wicklungsisolation von Motoren ohne Zerstörung unter Laborbedingungen realisiert werden kann. Die speziell angefertigte Testmaschine (1,4 MW ASM) mit Wicklungsanzapfungen ermöglicht das Einbringen einer zusätzlichen Kapazität um eine Veränderung der Gesamtkapazität und ihrer Eigenschaften zu ermöglichen.

Ausgehend von verfügbaren Studien in der Literatur, wird die Wicklungskapazität als der dominierende Parameter im Rahmen der Alterung identifiziert [3,4]. Als Richtwert für die Größenordnung der getesteten Kapazitätswerte wurde die identifizierte Wicklungskapazität der Maschine von je 21nF, gemessen von Phase zu Erde bzw. die aus weiteren Versuchen identifizierten prozentuellen Veränderungen herangezogen. Letztgenannte Versuche wurden an Formetten - Nutmodelle mit allen Isolationsmaterialien welche auch in der Serienmaschine enthalten sind, durchgeführt und werden in Abschn. 4 beschrieben

Die Indikatorwerte sind auf den Wert der Streuung der bei Aufnahme der Initialmessung durch Abweichungen der Einzelmessungen entsteht bezogen. Ein Wert des Isolationszustandsindikators von "1" entspricht dabei dem gesunden unveränderten Zustand der Maschine. Werte über "1" deuten auf eine Verschlechterung der Isolation hin. Die Ergebnisse zeigen bei steigender Kapazitäts- bzw. Impedanzänderung, was wiederum einer kontinuierlichen Isolationszustandsänderung der Maschine entspricht, eine monoton ansteigende Veränderung der Indikatorwerte. Der Nachweis der Monotonie ist besonders wichtig für einen späteren industriellen Einsatz.

Ein wichtiger Punkt für die praktische Anwendung des Diagnoseverfahrens ist dabei die Sensitivität des Fehlerindikators. Dazu muss auch die Streuung berücksichtigt werden um eine Abweichung vom Initialzustand beurteilen zu können. Hierzu dient die Darstellung der Einzelindikatoren in der sogenannten „Boxplot" Darstellung, cf. Abb. 6.

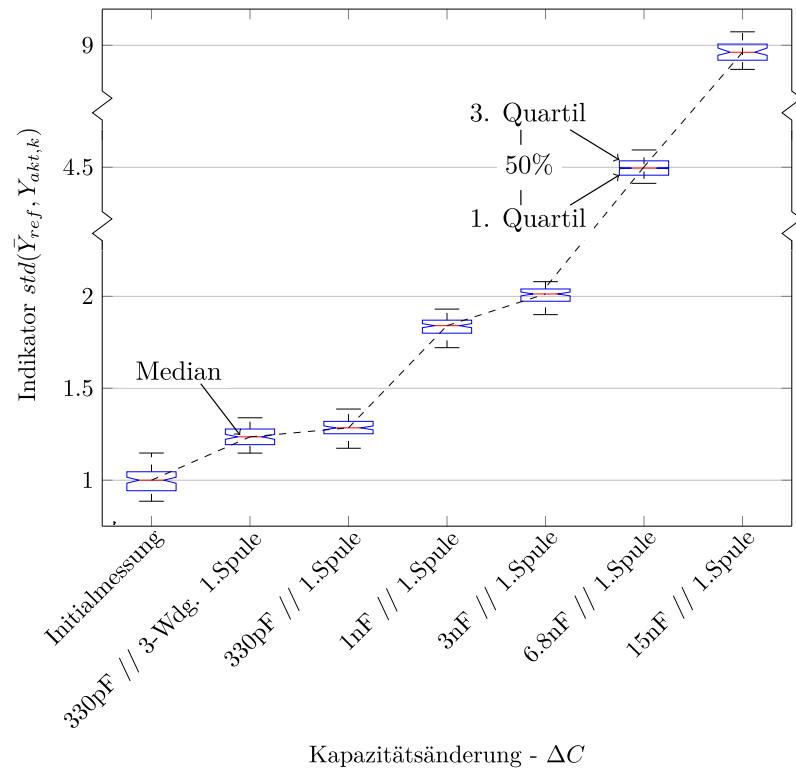

Abb. 6. Boxplot Darstellung der Messwerte aller getesteten Szenarien aus Abb. 5

Mit der "Boxplot" Darstellung ist eine statistische Aussage über die Verteilung der Einzelindikatoren möglich. Innerhalb der blauen Box befinden sich $50 \%$ der Werte. Die rote Linie stellt den Median des Datensatzes dar und damit die Mitte der Messwertreihe. Die deutliche Anhebung der Medianwerte und die geringe Streuung der Einzelmessungen impliziert, dass alle getesteten Szenarien sich deutlich vom Initialzustand unterscheiden.

Im folgenden Teil der Arbeit sollen Ergebnisse zum Thema beschleunigte Alterung von Isolationssystemen zeigen, wie sich Kapazitätswerte während der Alterung verändern.

\section{Verifikation der Isolationseigenschaften - beschleunigte Alterung}

Zusätzlich zu den hier vorgestellten Untersuchungen an der 1,4 MW Asynchronmaschine wurde die Änderung der Isolationsfestigkeit an speziell dafür angefertigten Statornutmodellen, im weiteren Verlauf als Formette bezeichnet, untersucht. Die Formetten wurden mit beschleunigten Alterungszyklen belastet, bis der Ausfall der Isolierung durch Spannungsbelastungstests erfolgt. Mit zusätzlichen zerstörungsfreien nichtinvasiven Diagnosemethoden, z. B. Dissipationsfaktor $(\tan \delta)$, Kapazität (C), soll die Korrelation zwischen diesen Indikatorwerten, die Ergebnisse des vorgeschlagenen Verfahrens und die Verschlechterung der Isolationsstärke bis zu einem Ausfall untersucht werden. In [5] wird eine Methode zur Identifizierung der elektrischen Alterung von Wicklungsisolationssystemen vorgestellt.

Einleitend soll hier der Begriff Alterung zuerst definiert werden. Alle Prozesse die eine irreversible Änderung im Isolationssystem bzw. in Teilen davon hervorrufen, welche zu einer Reduzierung der dielektrischen Eigenschaften führt, werden als Alterung verstanden. Veränderungen, welche als reversibel eingestuft werden, können aber trotzdem die dielektrischen Eigenschaften wesentlich beeinflussen oder sogar zu einem Ausfall führen, werden jedoch nur indirekt als Folge der Alterung angesehen. Darunter können die Einflüsse von Umgebungsbedingungen zählen, wie etwa Feuchtigkeit. Die Absorption von Wasser verschiedener Isolationsmaterialien kann zu einer deutlichen Änderung der dielektrischen Eigenschaften führen.

Aus Kostengründen erfolgen die Untersuchungen der Isolationsbeständigkeit nicht mit einer kompletten Maschine, sondern mit For- 


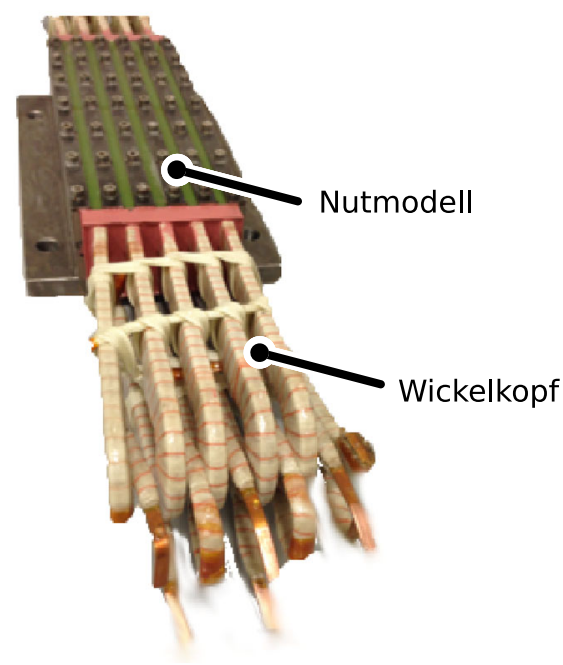

Abb. 7. Statornutmodell - Formette des Isolationsystems der ASM mit einer Leistung von 1,4 MW

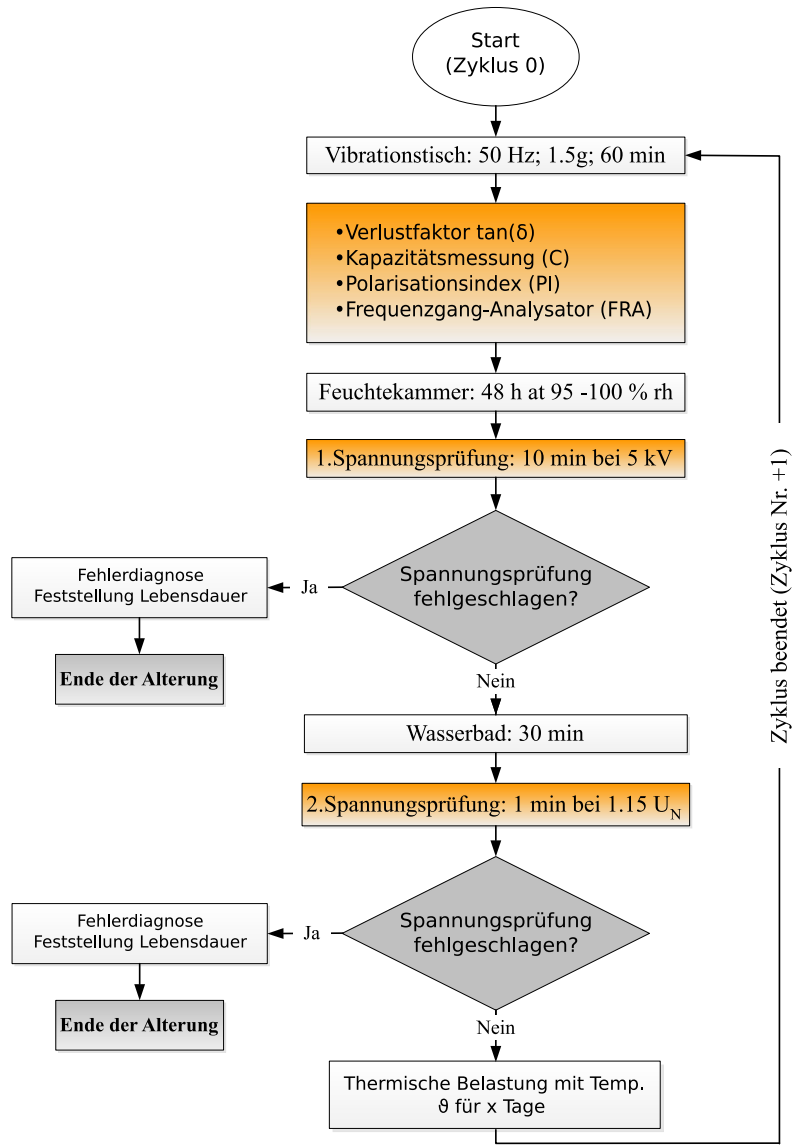

Abb. 8. Flussdiagramm des Alterungszyklus

metten, siehe Abb. 7. Die Isolationsmaterialien sind ident zur Serienmaschine, jedoch ist die Spulenweite aus Gründen der Platzeinsparung auf eine Nut verkürzt. Genau wie in der Maschine gibt es eine Ober- und Unterschicht in jeder Nut, wobei die jeweils äußersten Nuten mit einer halben Teilspule bestückt sind um alle Positionen

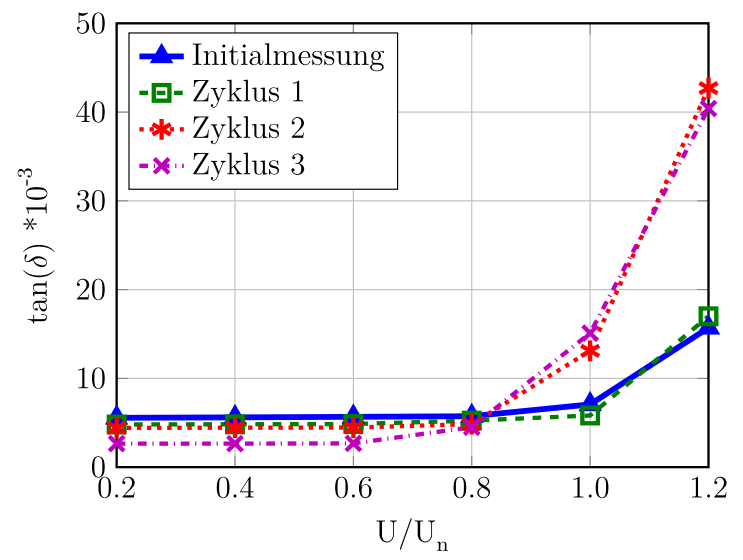

Abb. 9. Verlustfaktormessung des Isolationssystems an einer Formette

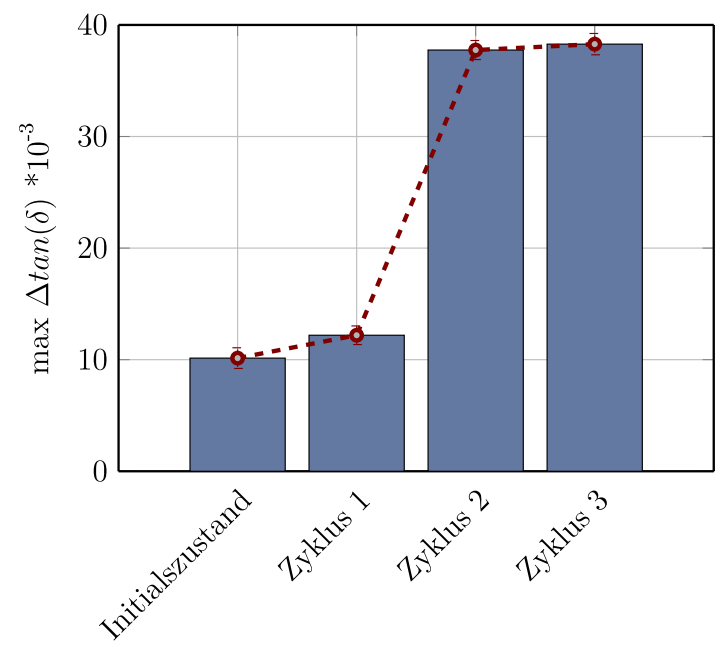

Abb. 10. $\Delta \tan \delta$-Werte als Betragsdifferenz zwischen $0.2 U_{n}$ und $1.2 U_{n}$

auszufüllen. Das Modell in Abb. 7 besitzt alle wesentlichen Eigenschaften eines realen Wicklungssystems.

Der angewendete Alterungszyklus ist nach Norm IEEE 1776 definiert. Jeder einzelne Zyklus besteht aus mehreren Stressbelastungen. Darunter zählen die thermische und mechanische Belastung in Form von Wärmelagerung, Rütteltisch sowie FeuchtekammerMasserbad und Spannungsprüfung. Die Belastung durch erhöhte Temperatur stellt die Hauptalterungsursache dar. Das Wasserbad ist laut Norm für gekapselte Isolationssysteme vorgesehen, wurde aber auch für das Zielsystem als Kriterium definiert. Die Spannungsprüfung dient als Kriterium um einen Ausfall zu definieren. In Abb. 8 ist der Durchlauf eines Alterungs- bzw. Prüfzyklus dargestellt. Insgesamt ist vorgesehen, dass die Formette eine Anzahl von etwa 10 Zyklen erreicht, bevor ein Ausfall erfolgt.

In Abb. 9 sind die Ergebnisse der ersten 3 Zyklen der Verlustfaktormessung dargestellt. Diese Messungen dienen dazu, eine schlüssige und aussagekräftige Bewertung des Isolationszustandes zu ermöglichen. Durch diese Messungen lassen sich Fehler bei der Imprägnierung für harzartige Systeme, Gaseinschlüsse, sowie Schäden durch Teilentladungen (TE) oder Delamination aufgrund von thermischer Beanspruchung feststellen. Der Verlustfaktor quantifiziert die dielektrischen Verluste in der Wicklungsisolierung. Während die 


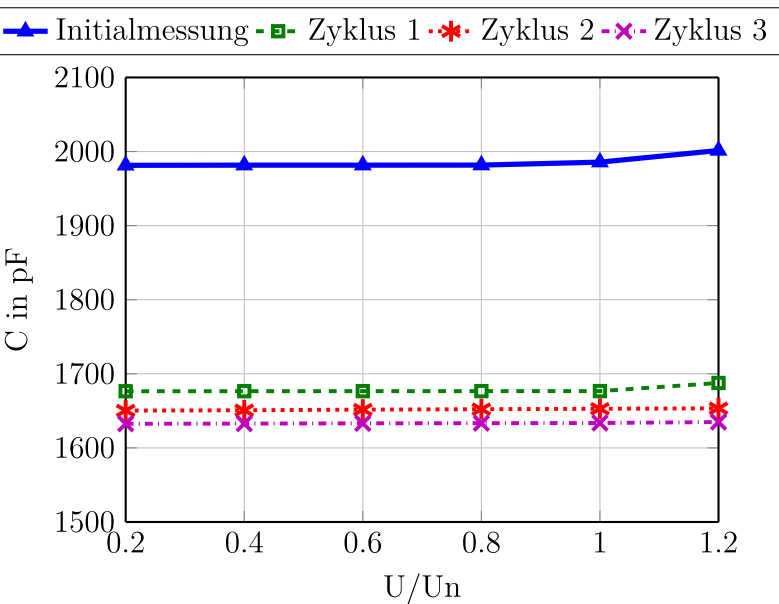

Abb. 11. Verlauf der Isolationskapazität bei jeweiligen Alterungszyklen

dielektrischen Verluste, und damit der Verlustfaktor, unterhalb der TE-Einsatzspannung durch Absorptionsverluste verursacht werden, beinhaltet der Verlustfaktor bei $U_{n}$ auch den Beitrag der lonisationsverluste durch Teilentladungen innerhalb der Hauptisolierung. Die zusätzlichen lonisationsverluste führen zu einem schnelleren Anstieg des Verlustfaktors oberhalb der TE-Einsatzspannung, wie deutlich in Abb. 9 zu erkennen ist. In [6] werden weitere Ergebnisse der Versuche präsentiert.

Um die Intensität von Teilentladungen zu beurteilen, wird die Steigung des Verlustfaktors in Relation zur Prüfspannung betrachtet, der sogenannte Delta-tan $\delta(\Delta \tan \delta)$. Je schneller der Verlustfaktor oberhalb der TE-Einsatzspannung ansteigt, desto höher ist das Niveau der Teilentladungen. In Abb. 10 sind die ansteigenden $\Delta \tan (\delta)$ Werte dargestellt als Veränderung vom Verlustfaktor bei Spannungsniveau $0.2 U_{n}$ und $1.2 U_{n}$. Die hohen Werte deuten auf eine bereits deutliche Verschlechterung der Isolationsfestigkeit hin.

Neben dem Verlustfaktor ist die Kapazität der Wicklungsanordnung von Bedeutung. Abbildung 11 zeigt die ermittelten Kapazitätswerte bei den unterschiedlichen Zyklen. Mit der Kapazität des aktuellen Zyklus $C_{x}$ und dem Anfangszustand $C_{0}$ lässt sich eine Änderungsrate $\Delta C$ berechnen zu $\Delta C=\left(C_{x}-C_{0}\right) / C_{0}$. Nach dem 3 . Alterungszyklus ist bereits eine Reduzierung der Wicklungskapazität von etwa $25 \%$ erreicht worden.

Die Isolationskapazität ist ein Parameter, der sich deutlich während der Alterung des Wicklungssystems ändert. Die Änderung der Kapazität geht einher mit einer Veränderung der hochfrequenten elektrischen Eigenschaften, speziell bei Sprunganregung und dieser Effekt wird bei der Zustandsüberwachungsmethode der Isolation genutzt.

Das Prinzip der Isolationszustandsüberwachung wurde ebenfalls während des Alterungsprozesses an den Formetten getestet und mit den vorher beschriebenen Diagnoseverfahren verglichen. Die Spektren der aufgezeichneten Stromtransienten während der fortschreitenden Alterung der Formetten sind in Abb. 12 dargestellt.

Die durchgezogene Kurve, mit ,Initialmessung' bezeichnet, zeigt den spektralen Verlauf für den anfänglichen unveränderten Zustand einer Formette. Der Zustand ,Zyklus 1' nach dem ersten Alterungszyklus zeigt einen nur leicht abweichenden Verlauf im Vergleich zur Initialmessung. Eine detektierbare Verschiebung findet erst bei höheren Resonanzen im Frequenzbereich statt. Der ,Zyklus 2' bzw. 'Zyklus 3' zeigt deutlich veränderte Resonanzerscheinungen. Nach den letzten beiden genannten Alterungszyklen ändern sich die parasitären Wicklungskapazitäten derart, dass eine deutliche Abweichung der spektralen Anteile eintritt und der daraus berechnete Indikatorwert deutlich ansteigt.

\section{Zusammenfassung}

Eine Schwächung der Wicklungsisolation korreliert mit einer Veränderung der zugehörigen transienten Isolationskapazität und zwangsläufig verkoppelt damit ergibt sich eine Veränderung des transienten elektrischen Hochfrequenzverhaltens der elektrischen Maschine. Das in dieser Veröffentlichung vorgestellte Verfahren zur Isolationszustandsüberwachung nutzt diese Zusammenhänge. Die transienten Stromantworten nach einer Schalthandlung des Wechselrichters werden erfasst und in weiterer Folge ein Isolationszustandsindikator ermittelt. Die in dieser Arbeit präsentierten Untersuchungsergebnisse wurden einerseits an einem Hochleistungstraktionsmotor (4-polige ASM; 1,4 MW, Formspulen) sowie andererseits an, entsprechend den Isolationsnormen abgeleiteten, Motoretten speziell für die Alterungsmessungen durchgeführt. Zusammenfassend kann festgehalten werden, dass die vorgestellte Analysemethode zur online Überwachung der Wicklungsisolation in der Lage ist, bereits eine beginnende Verschlechterung des Isolationszustandes der Statorwicklung (vor dem Auftreten eines Kurzschlusses in der Wicklung) zu erkennen. Dadurch kann die Wahrscheinlichkeit für einen unerwarteten Ausfall des Antriebs durch Schäden in der Isolation deutlich reduziert werden. Dies führt zu einer maßgeblichen Erhöhung der Zuverlässigkeit von elektrischen Antrieben und ermöglicht die Planung strategischer Wartung (CMB- Condition based maintenance).

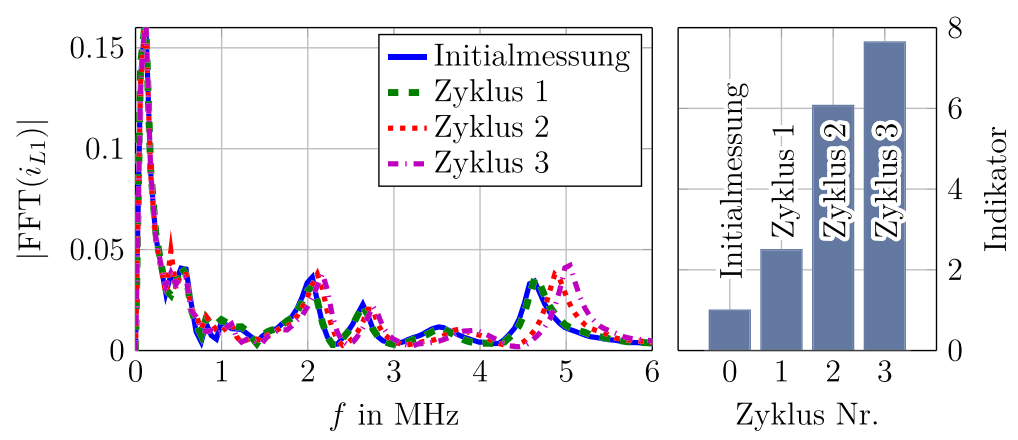

Abb. 12. Spektren der gemessenen Stromantworten an Formetten bei unterschiedlichem Alterungsfortschritt (links) und berechnete Indikatoren (rechts) 


\section{Danksagung}

Open access funding provided by TU Wien (TUW).

Die Arbeiten zu diesem Beitrag wurden durch Bombardier Transportation/BT-Rolling Stock Equipment (RoQ) unterstützt. Die Autoren dieser Veröffentlichung bedanken sich bei Herrn Dipl.-Ing. H. Mannsbarth (Head of RoQ_Mechanical Engineering), Herrn Dr.-ETH C. Gerster (Head of Engineering RoQ_Energy \& Motion) sowie Herrn Dipl.-Ing. M. Bazant (Head of RoQ_M_Drives Product Management \& Development and R\&D) für die große Unterstützung und das Projektfunding. Weiterer Dank geht an Bombardier Transportation Switzerland Ltd., insbesondere Herrn Dipl.-Ing.-ETH M. Jörg (Head of RoQ_Energy \& Motion; Product Engine HPP) in Zürich sowie an Herrn Dipl.-Ing. E. Moser (RoQ_MA_Drives Wien). Den Unternehmen ISOVOLTA-Austria (insbesondere Herrn Dipl.-Ing. W. Grubelnik sowie Dipl.-Ing. R. Fasching) sowie LEM-Corp. (Herrn Dr. W. Teppan und Herrn Dipl.-Ing. J. Burk) gilt es für die Kooperation zu danken. Diese Arbeit wurde gefördert von der österreichischen Forschungsförderungsgesellschaft (FFG) unter der Projektnummer 838478.

Open Access This article is distributed under the terms of the Creative Commons Attribution 4.0 International License (http://creativecommons.org/

\section{Autoren}

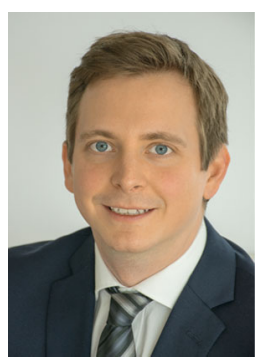

\section{Clemens Zöller}

received the Dipl.-Ing. and Dr. techn. (Ph.D.) degrees (with honor) in electrical engineering from Vienna University of Technology, Vienna, Austria, in 2013 and 2017, respectively. $\mathrm{He}$ is currently a research and teaching assistant with the Institute of Energy Systems and Electrical Drives, Vienna University of Technology, where he is engaged in several industrial and scientific projects with focus on electrical drives. His special fields of interest are fault detection, condition monitoring and sensorless control of inverter-fed AC machines.

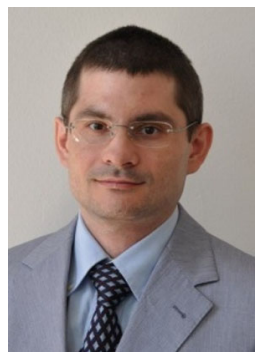

\section{Markus A. Vogelsberger}

received the Dipl.-Ing. (with honor) and Dr. techn. (Ph.D.) degrees (with honor) in electrical engineering from Vienna University of Technology, Vienna, Austria, in 2004 and 2009, respectively. He was a scientific research assistant at the Institute of Electrical Drives and Machines, TU Vienna, where he was engaged in several industrial and scientific R\&D projects. He joined Georgia Institute of Technology University, Atlanta/USA for study and research activities in 2006 and 2008, respectively. Since 2011, he has been with licenses/by/4.0/), which permits unrestricted use, distribution, and reproduction in any medium, provided you give appropriate credit to the original author(s) and the source, provide a link to the Creative Commons license, and indicate if changes were made.

\section{Literatur}

1. Mahdavi, S., Hameyer, K. (2012): High frequency equivalent circuit model of the stato winding in electrical machines. In XXth international conference on electrical machines, Marseille (S. 1706-1711).

2. Schröder, D. (2012): Leistungselektronische schaltungen; funktion, auslegung und anwendung. Berlin: Springer.

3. Stone, G. C., Boulter, E. E., Culbert, I., Dhirani, H. (2004). Electrical insuation for rotating machines, New York: IEEE Press.

4. Farahani, M., Gockenbach, E., Borsi, H., Schaefer, K., Kaufhold, M. (2010): Behavior of machine insulation systems subjected to accelerated thermal aging test. IEEE Trans. Dielectr. Electr. Insul., 17(5), 1364-1372.

5. Ruf, A., Paustenbach, J., Franck, D., Hameyer, K. (2017): A methodology to identify electrical ageing of winding insulation systems. In 2017 IEEE international electric machines and drives conference, IEMDC, Miami, FL.

6. Zoeller, C., Vogelsberger, M. A., Fasching, R., Grubelnik, W., Wolbank, Th. M. (2017) Evaluation and current-response-based identification of insulation degradation for high utilized electrical machines in railway application. IEEE Trans. Ind. Appl., 53(3), 1-12.

Bombardier Transportation, Vienna, Austria, as R\&D Project lead in Rolling Stock Equipment (RoQ)-Drives Engineering Department. His fields of interest include traction motors, power electronics, drives topics and multidisciplinary R\&D-Projects. In particular, currently his focus as responsible R\&D-Project Lead for Drives is on the research and managing of the high innovative condition monitoring (OIM) and speed sensorless drives (RSD) strategies for ac traction drives, and on the interdisciplinary \& multi objective R\&D-Project thermo efficient traction.

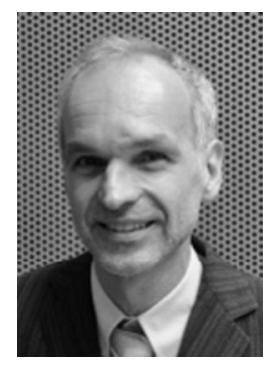

\section{Thomas M. Wolbank}

(M'1999) studied industrial electronics at the Vienna University of Technology, Vienna, Austria, where he also received the doctoral degree in 1996 and the Habilitation in 2004, respectively. At present he is with the Department of Energy Systems and Electrical Drives at the Vienna University of Technology. His research interests include saliency-based sensorless control of ac drives, dynamic properties and condition monitoring of inverter fed machines, transient electrical behavior of ac machines. He has co-authored some 150 papers in refereed journals and international conferences. 